\title{
Deciphering Molecular Mechanism Underlying Hypolipidemic Activity of Echinocystic Acid
}

\author{
Li Han, Peng Lai, and Jun-Rong Du \\ Department of Pharmacology, Key Laboratory of Drug Targeting and Drug Delivery Systems, Ministry of Education, \\ West China School of Pharmacy and Translational Neuroscience Center, Sichuan University, Chengdu 610041, China
}

Correspondence should be addressed to Jun-Rong Du; dujr07@gmail.com

Received 21 November 2013; Accepted 31 December 2013; Published 11 February 2014

Academic Editor: Firdous Jahan

Copyright (C) $2014 \mathrm{Li} \mathrm{Han}$ et al. This is an open access article distributed under the Creative Commons Attribution License, which permits unrestricted use, distribution, and reproduction in any medium, provided the original work is properly cited.

Our previous study showed that a triterpene mixture, consisting of echinocystic acid (EA) and oleanolic acid (OA) at a ratio of $4: 1$, dose-dependently ameliorated the hyperlipidemia and atherosclerosis in rabbits fed with high fat/high cholesterol diets. This study was aimed at exploring the mechanisms underlying antihyperlipidemic effect of EA. Molecular docking simulation of EA was performed using Molegro Virtual Docker (version: 4.3.0) to investigate the potential targets related to lipid metabolism. Based on the molecular docking information, isotope labeling method or spectrophotometry was applied to examine the effect of EA on the activity of 3-hydroxy-3-methylglutaryl coenzyme A (HMG-CoA) reductase, acyl-CoA:cholesterol acyltransferase (ACAT), and diacylglycerol acyltransferase (DGAT) in rat liver microsomes. Our results revealed a strong affinity of EA towards ACAT and DGAT in molecular docking analysis, while low binding affinity existed between EA and HMG-CoA reductase as well as between EA and cholesteryl ester transfer protein. Consistent with the results of molecular docking, in vitro enzyme activity assays showed that EA inhibited ACAT and DGAT, with $\mathrm{IC}_{50}$ values of 103 and $139 \mu \mathrm{M}$, respectively, and exhibited no significant effect on HMGCoA reductase activity. The present findings suggest that EA may exert hypolipidemic effect by inhibiting the activity of ACAT and DGAT.

\section{Introduction}

Hyperlipidemia is a key pathogenic factor for the development of cardiovascular and cerebrovascular diseases, such as atherosclerosis, hypertension, coronary heart disease, and brain stroke [1]. Pharmacotherapy is the primary way of ameliorating hyperlipidemia, among which statins and fibrate derivates are the most commonly used cholesteroland triglyceride-lowering drugs [2]. However, a substantial number of patients treated with these lipid-lowering drugs fail to effectively improve dyslipidemia [3]. Moreover, several adverse effects such as hepatic dysfunction and muscle injury are reported with statin and fibrate therapy $[4,5]$. In the recent years, with the advent of novel treatment targets for hyperlipidemia, numerous researches have been carried out in order to develop effective and safe lipid-lowering agents from natural products and synthetic compounds.

Phytochemical and pharmacological studies have demonstrated that triterpenoidal saponins are the main active constitutes of G. sinensis [6]. Our previous study demonstrated that oral administration of a pentacyclic triterpene mixture isolated from G. sinensis fruits (6 or $12 \mathrm{mg} / \mathrm{kg} /$ day), consisting of echinocystic acid (EA) and oleanolic acid (OA) at a ratio of $4: 1$, effectively improved the hyperlipidemia and subsequent atherosclerosis in rabbits fed with high fat/high cholesterol diets, suggesting the main constitute EA might be responsive to the hypolipidemic effect of triterpene extract from G. sinensis fruits in vivo [7]. It is reported that OA significantly inhibited the diacylglycerol acyltransferase (DGAT) from rat liver microsomes, lowered plasma cholesterol by inhibiting intestinal acyl-CoA:cholesterol acyltransferase (ACAT) activity in high-fat-fed hamsters, and protected against isoproterenolinduced myocardial ischemia in rats via antihyperlipidemic, antioxidative, and antiarrhythmic properties as well as its membrane-stabilizing action [8-10]. In addition, EA isolated from $G$. sinensis fruits prevented rat acute myocardial ischemia induced by isoproterenol and vasopressin [11]. 
Collectively, pentacyclic triterpenes, such as OA and EA, show potential therapeutic effects for cardiovascular diseases associated with dyslipidemia. However, the molecular mechanisms underlying the antihyperlipidemic effect of EA largely remain unclear.

A large body of studies has demonstrated that lipid profiles are governed by various enzymes and proteins, such as 3-hydroxy-3-methylglutaryl coenzyme A (HMG-CoA) reductase, cholesteryl ester transfer protein (CETP), ACAT, and DGAT. HMG-CoA reductase acts as the rate-limiting enzyme of cholesterol biosynthesis pathway by catalyzing the conversion of HMG to mevalonate [4]. CETP promotes the transfer of cholesteryl esters from antiatherogenic highdensity lipoprotein (HDL) to proatherogenic lipoproteins such as very low-density lipoprotein (VLDL) and lowdensity lipoprotein (LDL), and CETP inhibition or deficiency can effectively retard atherogenesis by increasing HDL and decreasing LDL [12]. ACAT catalyzes cholesterol esterification from cholesterol and fatty acyl coenzyme A, followed by subsequent cholesterol absorption, whereas ACAT inhibition is a therapeutic strategy for hypercholesterolemia and atherosclerosis through lowering cholesterol levels, diminishing the assembly and secretion of apolipoprotein Bcontaining lipoproteins such as VLDL, and inhibiting the formation of foam cells in the arterial walls [13]. DGAT catalyzes the formation of triglyceride in the final step of triglyceride biosynthesis via covalently linking a fatty acyl CoA with the free hydroxyl group of diacylglycerol, and DGAT inhibition is beneficial for the treatment of hypertriglyceridemia via decreasing serum triglyceride levels [14, 15]. Therefore, in order to explore the potential mechanisms of antihyperlipidemic effect of EA, the present study first performed the molecular docking of EA with HMG-CoA reductase, CETP, ACAT, and DGAT to predict the potential targets, and further investigated the effects of EA on the possible targets in in vitro rat liver microsomes.

\section{Materials and Methods}

2.1. Materials. Tris, phosphatidylserine, (R.S)-3-hydroxy3-methylglutaryl coenzyme A [(R.S)-HMG-CoA], nicotinamide adenine dinucleotide phosphate (NADPH), 1,2glyceryl dioleate, lecithin, and 3-oleic acid glycerol were purchased from Sigma-Aldrich (St. Louis, MO, USA). $\left[1-{ }^{14} \mathrm{C}\right]$ oleoyl-CoA was purchased from New England Nuclear Corporation (Boston, USA). Scintillation solution was purchased from Lipoluma, Lumac Co (Clanton, USA). BCA protein assay kit was from Boster Biological Technology (Wuhan, China). Pravastatin was obtained from Bristol-Myers Squibb (Shanghai, China). Other reagents were obtained from commercial sources.

2.2. Isolation of Echinocystic Acid (EA). The fruits of Gleditsia sinensis Lam. (G. sinensis) were collected from Sichuan province in China, and the aqueous extract was prepared as we described previously [7], followed by isolation of echinocystic acid (EA) using high-performance liquid chromatography (HPLC). In brief, chromatographic separation was conducted on a column $(40 \times 500 \mathrm{~mm})$ filled with reverse phase $\mathrm{C} 18$ silica gel. The mobile phase $\left(\mathrm{MeOH}-\mathrm{H}_{2} \mathrm{O}\right.$, $8: 2, v: v)$ was conveyed to the column at a flow rate of $10 \mathrm{~mL} / \mathrm{min}$ and the eluate was detected at $215 \mathrm{~nm}$ by diode array detector. EA $((3 \beta, 16 \alpha)$-3,16-dihydroxyolean-12-en-28oic acid, Figure 1) was collected and identified by spectral techniques including ${ }^{1} \mathrm{H}-\mathrm{NMR}$ and ESI-MS and the purity of EA was examined based on the percentage of total peak areas by HPLC. In the present study, EA (purity > 98\%) stock solution was prepared via mixing thoroughly $10 \mu \mathrm{L}$ Tween 80 , $20 \mu \mathrm{L}$ polyethylene glycol 200 , and $100 \mu \mathrm{L}$ water with $1 \mathrm{mg}$ EA and diluted with water prior to enzyme activity assay.

2.3. Molecular Docking Analysis. Molecular docking was performed by Molegro Virtual Docker (MVD) 4.3.0 tool adopting MolDock SE algorithm and Rerank scoring function as described previously [16]. The crystal structures of docked receptors (HMG-CoA reductase (PDB ID:1R31), CETP (PDB ID:2OBD), ACAT (PDB ID:1WL4), and DGAT (PDB ID: $1 \mathrm{~K} 30)$ ) were retrieved from RCSB Protein Data bank (http://www.rcsb.org/). The crystal structure of EA as docked ligand was available from NCBI's PCCompound database (http://pubchem.ncbi.nlm.nih.gov/).

Before docking, the receptor protein imported into the MVD software was preprocessed by removing cofactor, crystal water, and initial ligand, and then the free receptor protein was modified by adding the surface which shows the charge distribution of the receptor protein. Following the import of ligand EA into the MVD, molecular docking simulation of EA was performed to investigate the binding affinity of the receptor protein by computing the binding free energy between EA and the possible binding site of the receptor according to the software manual. The possible binding modes between EA and the ideal receptors were then analyzed according to the results of molecular docking. We conducted the docking of EA with HMG-CoA reductase, CETP, ACAT, and DGAT, receptively. The docking was done with the setting of the MVD as follows: (a) score: MolDock score [Grid]; (b) grid resolution (A): 0.30; (c) max iterations: 1500; (d) max population size: 50; (e) other parameters were the default setting. The pictures were prepared using MVD of 4.3.0 version.

\subsection{Preparation of Rat Liver Microsomes. Male SPF Sprague-} Dawley rats, $260 \sim 300 \mathrm{~g}$, were purchased from Chongqing Tengxin Animal Center (Chongqing, China). The procedure of animal experiment was carried out following the institutional guidelines of Animal Care and Use Committee at Sichuan University.

Rat liver microsomes were prepared as described previously $[17,18]$. In brief, the rats were killed by decapitation. Then, the liver was swiftly removed; rinsed by cold $0.9 \%$ $\mathrm{NaCl}$ solution; weighed,; cut finely into pieces with scissors; placed into 9 vol. of cold homogenization medium containing $137 \mathrm{mM} \mathrm{NaCl}, 2.7 \mathrm{mM} \mathrm{KCl}, 10 \mathrm{mM} \mathrm{Na} \mathrm{HPO}_{4}, 2 \mathrm{mM}$ $\mathrm{KH}_{2} \mathrm{PO}_{4}, 100 \mathrm{mM}$ sucrose, $10 \mathrm{mM}$ EDTA, and $2 \mathrm{mM}$ DTT; and homogenized in a Teflon homogenizer for $10 \mathrm{~min}$. The homogenate was subsequently centrifuged at $12000 \times \mathrm{g}$ for 


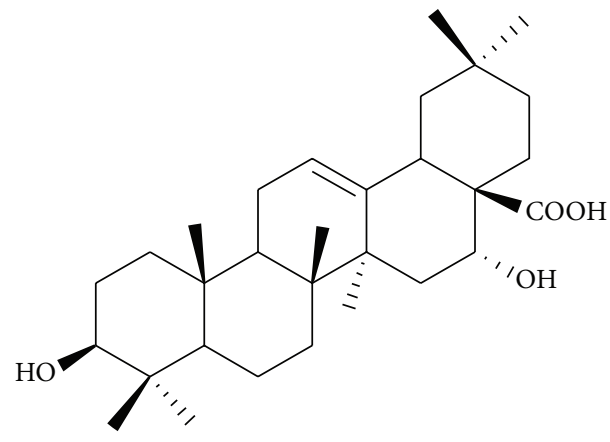

Figure 1: Chemical structure of echinocystic acid (EA).

$20 \mathrm{~min}$ at $4^{\circ} \mathrm{C}$ (Beckmann refrigerated centrifuge TJ-6). The supernatant fraction was sucked out and centrifuged again at $100000 \times \mathrm{g}$ for $60 \mathrm{~min}$ at $4^{\circ} \mathrm{C}$ after adding a certain amount of $1 \mathrm{M} \mathrm{CaCl}_{2}$ solution (final concentration of $\mathrm{CaCl}_{2}: 8 \mathrm{mM}$ ). The precipitation fraction (microsomes) was acquired via the removal of the supernatant fraction. The prepared microsomes were, respectively, resuspended in $\mathrm{KCl}$-Tris- $\mathrm{HCl}$ buffer solution (Tris- $\mathrm{HCl}: 10 \mathrm{mM}, \mathrm{KCl}: 100 \mathrm{mM}$, and $\mathrm{pH}$ 7.4) of $100-200 \mu \mathrm{L}$ or $2.5 \mathrm{M}$ sucrose solutions, mixed thoroughly, and then stored in $-80^{\circ} \mathrm{C}$ for assays of microsomal enzyme activities.

2.5. Assay of Microsomal HMG-CoA Reductase Activity. Effect of EA on HMG-CoA reductase activity was tested via spectrophotometry using HMG-CoA and cofactor NADPH as described previously [19]. Briefly, the reactive mixture containing $100 \mu \mathrm{L}$ of $0.2 \mathrm{mM}$ NADPH, $600 \mu \mathrm{L}$ of phosphate buffer ( $\mathrm{pH}$ 6.8) (composed of $300 \mathrm{mM} \mathrm{KCI,} 240 \mathrm{mM}$ potassium phosphate, $6 \mathrm{mM}$ EDTA, and $15 \mathrm{mM}$ DTT), $100 \mu \mathrm{L}$ of the prepared microsome suspension $(10 \mathrm{mg} / \mathrm{mL}$ protein), and $10 \mu \mathrm{L}$ of the test sample EA $(200 \mathrm{mM})$ or the positive control pravastatin $(50 \mathrm{mM})$ was first monitored at $340 \mathrm{~nm}$ using ultraviolet spectrophotometer (UNICO) for HMGCoA-independent oxidation of NADPH. The reaction was then initiated by adding $100 \mu \mathrm{L}$ of $1 \mathrm{mM}$ (R.S)-HMG-CoA. After $5 \mathrm{~min}$ of incubation at $37^{\circ} \mathrm{C}$, the supernatant was sucked out and tested at $340 \mathrm{~nm}$ by spectrophotometer for HMGCoA-dependent oxidation of NADPH. One unit of HMGCoA reductase was defined as the amount of enzyme that catalyzes the oxidation of $1 \mu \mathrm{mol}$ of NADPH per gram of microsome protein. The protein concentration was measured by the method of BCA using BSA as the standard. The inhibitory effect of EA or pravastatin was calculated as a percentage of HMG-CoA reductase activity of control group, respectively.

2.6. Assay of Microsomal ACAT Activity. Effect of EA on ACAT activity was tested by the isotope labeling method as reported previously [20]. In brief, the prepared microsomes were unfrozen and dissolved at $37^{\circ} \mathrm{C}$ water bath. The reactive mixture, containing $10 \mu \mathrm{L}$ of microsome suspension $(10 \mathrm{mg} / \mathrm{mL}$ protein), $20 \mu \mathrm{L}$ of $0.5 \mathrm{M}$ potassium phosphate buffer ( $\mathrm{pH} 7.4,10 \mathrm{mM}$ DTT), $10 \mu \mathrm{L}$ of BSA $(180 \mathrm{mg} / \mathrm{mL})$, $2.0 \mu \mathrm{L}$ of cholesterol in acetone $(20 \mathrm{mg} / \mathrm{mL}), 130 \mu \mathrm{L}$ of water, and $10 \mu \mathrm{L}$ of the test sample EA at a concentration range of $0 \sim 400 \mu \mathrm{M}$, was preincubated for $30 \mathrm{~min}$ at $37^{\circ} \mathrm{C}$. The reaction was started by adding $10 \mu \mathrm{L}$ of $\left[1-{ }^{14} \mathrm{C}\right]$ oleoyl-CoA $(0.05$ $\mu \mathrm{Ci}$ : final concentration $10 \mu \mathrm{M})$. After $30 \mathrm{~min}$ incubation at $37^{\circ} \mathrm{C}$, the reaction was terminated by adding $1.0 \mathrm{~mL}$ of $i$ $\mathrm{PrOH}-n$-hexane $(4: 1, v / v)$ solution. A mixture of $0.6 \mathrm{~mL}$ of $n$-hexane and $0.4 \mathrm{~mL}$ of $0.1 \mathrm{M}$ potassium phosphate buffer was subsequently added to the reaction mixture and mixed uniformly by vortexing. Standing for $2 \mathrm{~min}$ was allowed to separate the reaction mixture into aqueous and organic phases. The upper organic phase containing the radiolabeled cholesteryl ester products was sucked out. The radioactivity in $100 \mu \mathrm{L}$ of the upper phase was determined using $4 \mathrm{~mL}$ of scintillation cocktail (Lipoluma, Lumac Co.) by a LS6000 Beckman Liquid Scintillation Counter (Beckman Inc). Data were presented as counts per minute (CPM) of $\left[1-{ }^{14} \mathrm{C}\right]$ cholesteryl ester products and the readings were normalized to protein concentrations, which were measured by the method of BCA using BSA as the standard. Effect of EA on ACAT activity was calculated as the percentage of inhibition versus control group. Software Origin 7.5 (OriginLab, USA) was used to draw the relation curve of drug concentrations with the inhibition rate, and the $50 \%$ inhibitory concentration $\left(\mathrm{IC}_{50}\right)$ was calculated.

2.7. Assay of Microsomal DGAT Activity. Effect of EA on DGAT activity was tested by the isotope labeling method as reported previously [8]. In brief, EA at a concentration range of $0 \sim 400 \mu \mathrm{M}$ was incubated $30 \mathrm{~min}$ at $37^{\circ} \mathrm{C}$ with the prepared microsome suspension $(10 \mathrm{mg} / \mathrm{mL}$ protein $),\left[1-{ }^{14} \mathrm{C}\right]$ oleoylCoA $(0.05 \mu \mathrm{Ci}$ : final concentration $3 \mu \mathrm{M}), 3 \mathrm{mM}$ 1,2-glyceryl dioleate, and the incubation buffer that was composed of $200 \mu \mathrm{M} \mathrm{MgCl}_{2}, 1 \mathrm{mg} / \mathrm{mL}$ fatty acid-free BSA, $100 \mu \mathrm{M}$ lecithin, $100 \mu \mathrm{M}$ phosphatidylserine, and $5 \mathrm{mM}$ Tris- $\mathrm{HCl}(\mathrm{pH} \mathrm{8)}$. After $30 \mathrm{~min}$ of incubation, the reaction was stopped by adding chloroform-methanol $(1: 1, v / v)$ solution, chloroform and acidified sodium chloride solution (containing $17 \mathrm{mM}$ $\mathrm{NaCl}$ and $1 \mathrm{mM} \mathrm{H}_{2} \mathrm{SO}_{4}$ ). The precooled unlabelled glyceryl trioleate was added to the above reaction mixture. The lipids in the mixture were extracted into the organic solvent by centrifugation at $2500 \mathrm{rpm}$ for $10 \mathrm{~min}$. The lower organic phrase containing lipids was recovered, dried under nitrogen, and redissolved in $100 \mu \mathrm{L}$ of chloroform. The lipids were then separated by silica gel thin-layer chromatography plate in chloroform : diethyl ether : acetic acid $(9: 6: 4, v / v / v)$. Triglyceride-specific bands were scraped after being verified by standards with exposure to $\mathrm{I}_{2}$ vapor, and the radioactivity was measured by liquid scintillation counting. Data were presented as CPM of $\left[1-{ }^{14} \mathrm{C}\right]$ triglyceride products and the readings were normalized to protein concentrations. Effect of EA on DGAT activity was calculated as the percentage of inhibition versus control group. Software Origin 7.5 was used to draw the relation curve of drug concentrations with the inhibition rate, and the $\mathrm{IC}_{50}$ was calculated.

2.8. Statistical Analysis. Data are presented as mean \pm SD. Statistical analyses were performed with SPSS 16.0 software. The significance of data between the tested groups was 


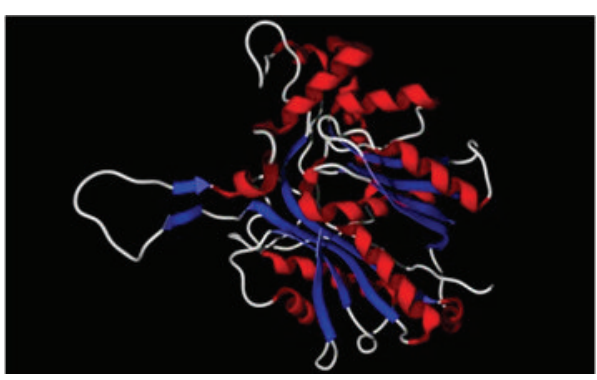

(a)

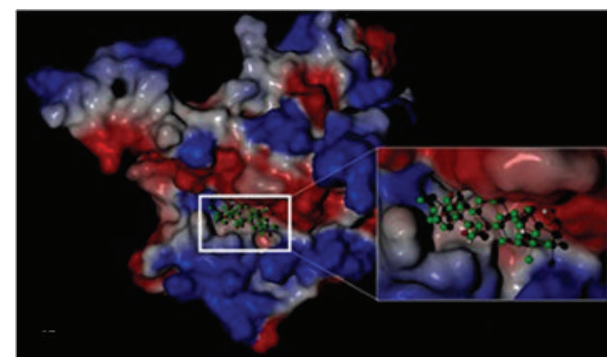

(b)

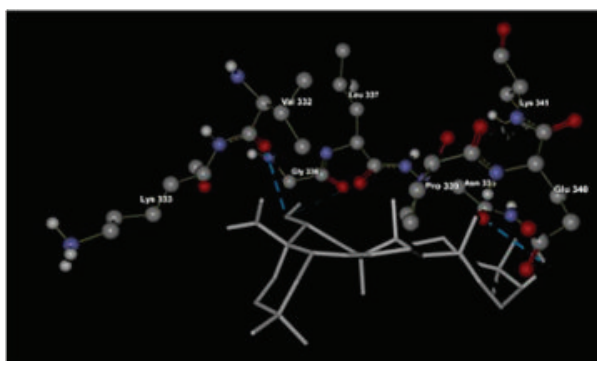

(c)

FIGURE 2: Molecular docking of echinocystic acid (EA) with ACAT in 3D diagram. (a) Three-dimensional structure of ACAT. (b) Optimized docking conformation of EA in the hydrophobic pocket of ACAT. The surface of ACAT was color-coded by electrostatic potential. Red, positive charge; white, neutral; blue, negative charge. (c) Detailed binding mode of EA with ACAT. Dotted blue lines display the hydrogen bonding between the carboxyl group and $\mathrm{OH}$ group of EA and amino acid residues Gly 336 and Glu 340 of ACAT.

determined by one-way ANOVA. The probability level for statistical significance was set at $P<0.05$.

\section{Results and Discussion}

3.1. Molecular Docking. To predict the potential targets of lipid-lowering effects of EA, we performed the molecular docking of EA with HMG-CoA reductase, DGAT, ACAT, and CETP by MVD 4.3.0 tool using Rerank scoring function, respectively. As shown in Table 1, Rerank scores were recorded and used as the index of binding free energy between the ligand and the receptor protein, which is known to be negatively correlated with binding affinity. The docking results showed that EA exhibited a relatively strong binding affinity with ACAT and DGAT as inferred by their negative Rerank scores, -53 and -41 , which indicate low binding free energy; the binding affinity between EA and HMGCoA reductase was found to be very low, so was the binding affinity between EA and CETP, as evidenced by their positive Rerank scores, +4 and +12 . These data indicate that EA has a strong binding affinity with ACAT and DGAT. In addition, it is reported that the negative free energy change $(\Delta G)$ values suggests a spontaneous interaction and correspond to a spontaneous binding process [21]. Therefore, the binding process between EA and HMG-CoA reductase and the binding between EA and CETP were probably not pontaneous, which implied that there was no specific binding ability of EA to HMG-CoA reductase and CETP. These molecular docking results suggest that ACAT and DGAT rather than HMG-CoA reductase and CETP are likely to be the potential targets of lipid-lowering effects of EA.
TABLE 1: Docking scores of echinocystic acid with the enzymes/ protein related to lipid metabolism.

\begin{tabular}{lc}
\hline Enzymes & Rerank score \\
\hline HMG-CoA reductase & +4 \\
CETP & +12 \\
ACAT & -53 \\
DGAT & -41 \\
\hline
\end{tabular}

Following the results mentioned above, we further analyzed the binding modes and interactions of EA with ACAT and DGAT, respectively. The results were shown in Figures 2 and 3. According to the results of MVD docking simulation, EA was most likely bound to ACAT's site within the hydrophobic pocket which is rich in hydrophobic amino acids such as Val, Leu, and Pro, as depicted in Figures 2(b) and 2(c). As a triterpenoid acid, EA has good hydrophobic property, which benefits from binding between the amino acid residues and small molecular compounds with hydrophobic property, so hydrophobic forces may well be one of the main interaction forces behind the binding of EA with ACAT. Furthermore, as seen in Figure 2(c), the OH group and carboxyl group of EA form two hydrogen bonds with residues Gly 336 and Glu 340 of ACAT, respectively. Therefore, the hydrogen bonds may be another interaction force behind the binding of EA and ACAT. As for DGAT, the MVD docking simulation revealed that it had a similar binding mode with EA towards ACAT. EA was also most probably going to bind with DGAT's site within the hydrophobic pocket (Figure 3(b)), which is likely due to the similar structure of DGAT to 


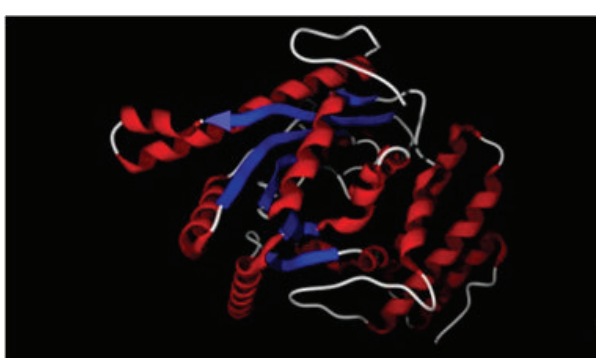

(a)

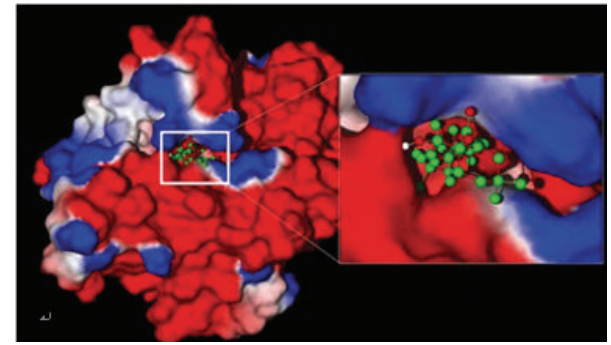

(b)

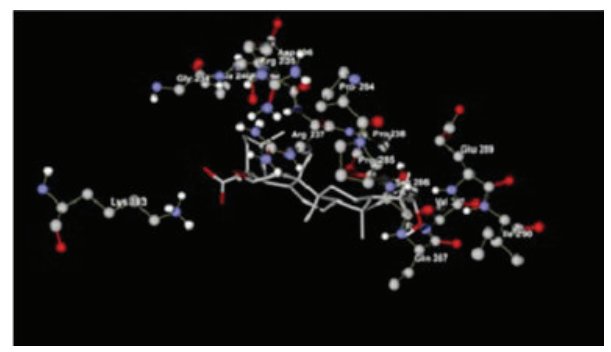

(c)

FIGURE 3: Molecular docking of echinocystic acid (EA) with DGAT in 3D diagram. (a) Three-dimensional structure of DGAT. (b) Optimized docking conformation of EA in the hydrophobic pocket of DGAT. The surface of DGAT was color-coded by electrostatic potential. Red, positive charge; white, neutral; blue, negative charge. (c) Detailed binding mode of EA with DGAT. Dotted blue line displays the hydrogen bonding between the carboxyl group of EA and residue Lys 193 of DGAT.

ACAT since both enzymes belong to the membrane-bound O-acyltransferase (MBOAT) family. However, the pocket, which is wealthy in residue Pro, was obviously not large enough to accommodate the whole structure of EA. It thus apparently decreases the hydrophobic interaction strength between EA and its surrounding residues, which, in turn, may decrease the inhibitory effect of EA on DGAT activity. Moreover, as shown in Figure 3(c), only one hydrogen bond was established between the carboxyl group of EA and residue Lys 193 of DGAT, which may be another reason why the binding affinity of EA with ACAT is stronger than that of EA with DGAT. These results are consistent with that obtained by Rerank scoring function which show that EA has a lower binding free energy and stronger binding affinity with ACAT compared to that with DGAT.

3.2. HMG-CoA Reductase Activity. HMG-CoA reductase is the rate-limiting enzyme of cholesterol biosynthesis pathway and thus is regarded as a major target for regulating hypercholesterolemia. In our previous study, 14-week treatment with a triterpene mixture consisting of $9.6 \mathrm{mg}$ EA and $2.4 \mathrm{mg}$ OA once daily (i.g.) decreased the total cholesterol levels in serum, aorta homogenates, and liver homogenates by $43 \%, 72 \%$, and $75 \%$, respectively, in hyperlipidemia and atherosclerosis rabbits fed with high fat/high cholesterol diets [7]. By contrast, however, the present molecular docking showed that the binding affinity between EA and HMG-CoA reductase was very low, suggesting that HMG-CoA reductase is not likely to be the potential target of cholesterol-lowering effect of EA. To validate this speculation, we assayed the effect of EA on the activity of HMG-CoA reductase in rat liver microsomes by spectrophotometry. As shown in Figure 4, EA showed no HMG-CoA reductase inhibitory activity even at $200 \mu \mathrm{M}(P>0.05)$, while pravastatin a concentration of $50 \mu \mathrm{M}$ exhibited a significant inhibition $(P<0.05)$ compared with controls. Taken together, the current results demonstrate that the other targets rather than HMG-CoA reductase is responsive to cholesterol-lowering activity of EA in vivo.

3.3. ACAT Activity. ACAT is regarded as a novel target for the treatment of hypercholesterolemia and atherosclerosis [13]. ACAT inhibitors, such as pactimibe, are reported to have cholesterol-lowering and antiatherosclerotic effects [22]. It is reported that $\mathrm{OA}$ at a concentration of $50 \mu \mathrm{M}$ significantly inhibited ACAT activity in Caco- 2 cells, a human intestinal cell line [9]. The present molecular docking showed that the binding affinity between EA and ACAT was much stronger than that between EA and HMG-CoA reductase, suggesting that ACAT is likely to be responsive for cholesterol-lowering effect of EA. Therefore, we investigated the effect of EA on ACAT activity in rat liver microsomes using the isotope labeling method. As shown in Figure 5, EA at a concentration range of $0 \sim 400 \mu \mathrm{M}$ concentration-dependently reduced ACAT activity, with an $\mathrm{IC}_{50}$ value of $103 \mu \mathrm{M}$, suggesting that ACAT inhibition contributes to the potent cholesterollowering effect of EA/OA mixture in vivo.

3.4. DGAT Activity. Hypertriglyceridemia is known as a major risk factor of obesity and cardiovascular diseases [23]. DGAT, a key enzyme in triacylglycerol synthesis, is regarded as a potential target for the treatment of triglyceride metabolic disorders $[14,15,24]$. OA is reported to significantly inhibit 


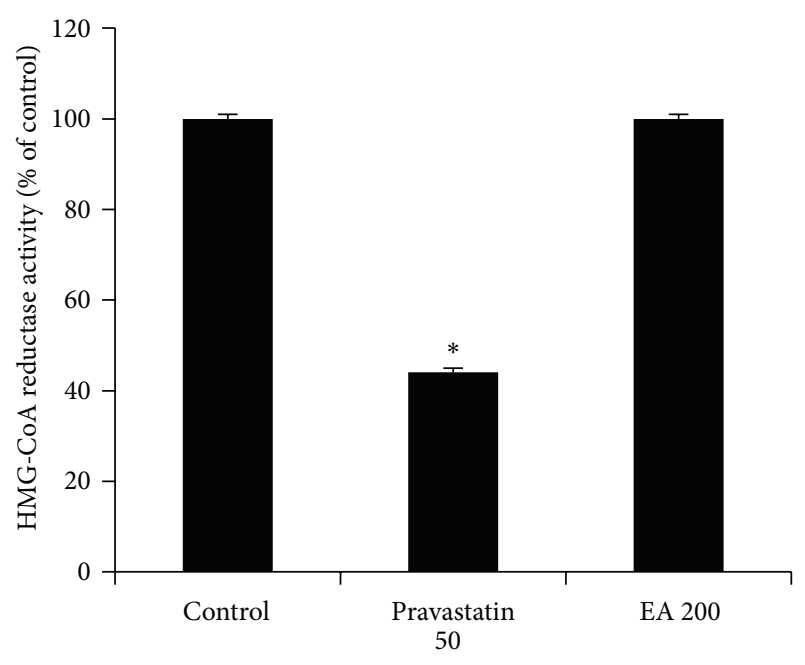

FIGURE 4: Effect of echinocystic acid (EA) on HMG-CoA reductase activity in rat liver microsomes. Pravastatin was used as a positive control. The inhibitory effect of $50 \mu \mathrm{M}$ pravastatin (pravastatin 50) or $200 \mu \mathrm{M}$ EA (EA 200) was calculated as the percentage of HMGCoA reductase activity of control group, respectively. Data are expressed as mean $\pm \mathrm{SD}(n=5) .{ }^{*} P<0.05$ versus control group, determined by one-way ANOVA.

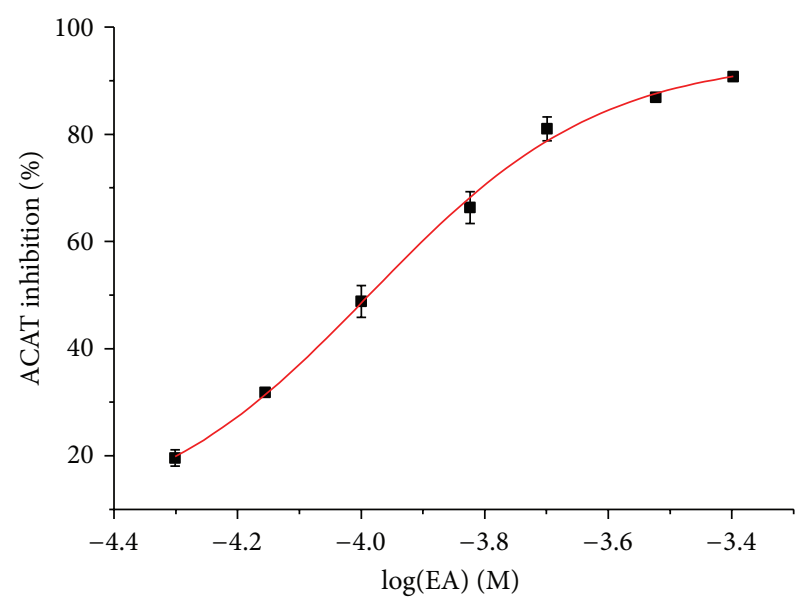

Figure 5: Effect of echinocystic acid (EA) on ACAT activity in rat liver microsomes. The inhibitory effect of EA was calculated as the percentage of inhibition versus control group. Data represent mean \pm SD of three independent experiments.

DGAT from rat liver microsomes [8]. In our previous study, 14-week treatment with a triterpene mixture consisting of $9.6 \mathrm{mg}$ EA and $2.4 \mathrm{mg} \mathrm{OA}$ once daily (i.g.) decreased the triacylglycerol levels in serum and aorta homogenates by $54.5 \%$ and $29 \%$, respectively, in hyperlipidemia and atherosclerosis rabbits fed with high fat/high cholesterol diets [7]. The molecular docking results showed that EA exhibited a relatively strong binding affinity with DGAT, suggesting that DGAT inhibition is probably associated with triacylglycerollowering effect of EA. Therefore, we investigated the effect of EA on DGAT activity in rat liver microsomes using the

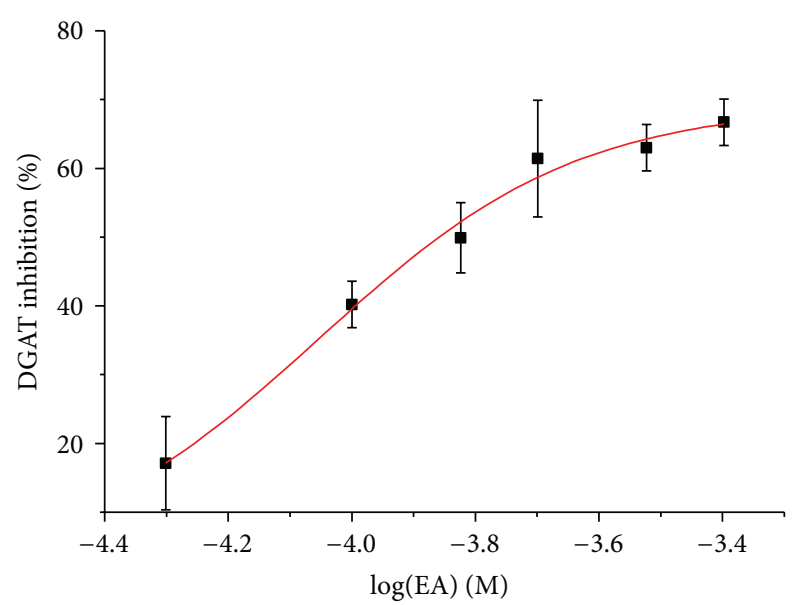

FIGURE 6: Effect of echinocystic acid (EA) on DGAT activity in rat liver microsomes. The inhibitory effect of EA was calculated as the percentage of inhibition versus control group. Data represent mean \pm SD of three independent experiments.

isotope labeling method. As shown in Figure 6, EA at a concentration range of $0 \sim 400 \mu \mathrm{M}$ concentration-dependently reduced DGAT activity in rat liver microsomes, with an $\mathrm{IC}_{50}$ value of $139 \mu \mathrm{M}$, suggesting that DGAT inhibition is responsive to triacylglycerol-lowering effect of EA/OA mixture in vivo.

Taken together, our current findings show, for the first time, that EA inhibits ACAT and DGAT with $\mathrm{IC}_{50}$ values of 103 and $139 \mu \mathrm{M}$, respectively, and exhibits no significant effect on the activity of HMG-CoA reductase. These results suggest that EA is a potential natural hypolipidemic agent by inhibiting ACAT and DGAT activity.

\section{Conflict of Interests}

The authors declare that there is no conflict of interests regarding the publication of this paper.

\section{Authors' Contribution}

Li Han and Peng Lai equally contributed to this study.

\section{Acknowledgments}

This work was supported in part by Sichuan University 985 Projects "Science and Technology Innovation Platform for Novel Drug Development" and "Translational Neuroscience Center." The authors are grateful to Dr. Xiaoping Gao for providing echinocystic acid.

\section{References}

[1] G. Veronica and R. R. Esther, "Aging, metabolic syndrome and the heart," Aging and Disease, vol. 3, no. 3, pp. 269-279, 2012.

[2] A. S. Wierzbicki, D. P. Mikhailidis, and R. Wray, "Drug treatment of combined hyperlipidemia," American Journal of Cardiovascular Drugs, vol. 1, no. 5, pp. 327-336, 2001. 
[3] T. A. Pearson, I. Laurora, H. Chu, and S. Kafonek, "The lipid treatment assessment project (L-TAP): a multicenter survey to evaluate the percentages of dyslipidemic patients receiving lipid-lowering therapy and achieving low-density lipoprotein cholesterol goals," Archives of Internal Medicine, vol. 160, no. 4, pp. 459-467, 2000.

[4] A. Kashani, C. O. Phillips, J. M. Foody et al., "Risks associated with statin therapy: a systematic overview of randomized clinical trials," Circulation, vol. 114, no. 25, pp. 2788-2797, 2006.

[5] A. Keech, R. J. Simes, P. Barter et al., "Effects of long-term fenofibrate therapy on cardiovascular events in 9795 people with type 2 diabetes mellitus (the FIELD study): randomized controlled trial," Lancet, vol. 366, no. 9500, pp. 1849-1861, 2005.

[6] J. H. Wang, J. Tang, D. Li, and L. G. Zhou, "Chemical constituents and bioactivity of Gleditsia plants," Chinese Wild Plant Resources, vol. 27, no. 6, pp. 1-3, 2008.

[7] P. Lai, J.-R. Du, M.-X. Zhang et al., "Aqueous extract of Gleditsia sinensis Lam. fruits improves serum and liver lipid profiles and attenuates atherosclerosis in rabbits fed a high-fat diet," Journal of Ethnopharmacology, vol. 137, no. 3, pp. 1061-1066, 2011.

[8] T. D. Nguyen, F. C. Xing, M.-C. Rho, S. L. Hyun, K. Bae, and H. K. Young, "The inhibition of diacylglycerol acyltransferase by terpenoids from Youngia koidzumiana," Archives of Pharmacal Research, vol. 28, no. 2, pp. 164-168, 2005.

[9] Y. Lin, M. A. Vermeer, and E. A. Trautwein, "Triterpenic acids present in hawthorn lower plasma cholesterol by inhibiting intestinal ACAT activity in hamsters," Evidence-Based Complementary and Alternative Medicine, vol. 2011, Article ID 801272, 9 pages, 2011.

[10] S. Senthil, M. Sridevi, and K. V. Pugalendi, "Cardioprotective effect of oleanolic acid on isoproterenol-induced myocardial ischemia in rats," Toxicologic pathology, vol. 35, no. 3, pp. 418423, 2007.

[11] J. Wu, J. Li, Z. Zhu et al., "Protective effects of echinocystic acid isolated from Gleditsia sinensis Lam. against acute myocardial ischemia," Fitoterapia, vol. 81, no. 1, pp. 8-10, 2010.

[12] P. J. Barter, H. B. Brewer Jr., M. J. Chapman, C. H. Hennekens, D. J. Rader, and A. R. Tall, "Cholesteryl ester transfer protein: A novel target for raising HDL and inhibiting atherosclerosis," Arteriosclerosis, Thrombosis, and Vascular Biology, vol. 23, no. 2, pp. 160-167, 2003.

[13] C. Leon, J. S. Hill, and K. M. Wasan, "Potential role of Acyl-coenzyme A:cholesterol transferase (ACAT) inhibitors as hypolipidemic and antiatherosclerosis drugs," Pharmaceutical Research, vol. 22, no. 10, pp. 1578-1588, 2005.

[14] A. J. King, J. A. Segreti, K. J. Larson et al., "Diacylglycerol acyltransferase 1 inhibition lowers serum triglycerides in the zucker fatty rat and the hyperlipidemic hamster," Journal of Pharmacology and Experimental Therapeutics, vol. 330, no. 2, pp. 526-531, 2009.

[15] C.-L. E. Yen, S. J. Stone, S. Koliwad, C. Harris, and R. V. Farese Jr., "DGAT enzymes and triacylglycerol biosynthesis," Journal of Lipid Research, vol. 49, no. 11, pp. 2283-2301, 2008.

[16] B. S. Jayashree, S. Thomas, and Y. Nayak, "Design and synthesis of 2-quinolones as antioxidants and antimicrobials: a rational approach," Medicinal Chemistry Research, vol. 19, no. 2, pp. 193209, 2010.

[17] M. S. Brown, J. L. Goldstein, and J. M. Dietschy, "Active and inactive forms of 3-hydroxy-3-methylglutaryl coenzyme A reductase in the liver of the rat. Comparison with the rate of cholesterol synthesis in different physiological states," Journal of Biological Chemistry, vol. 254, no. 12, pp. 5144-5149, 1979.
[18] G. C. Ness, C. E. Sample, and M. Smith, "Characteristics of rat liver microsomal 3-hydroxy-3-methylglutaryl-coenzyme A reductase," Biochemical Journal, vol. 233, no. 1, pp. 167-172, 1986.

[19] J. H. Sung, S.-J. Lee, K. H. Park, and T. W. Moon, "Isoflavones inhibit 3-hydroxy-3-methylglutaryl coenzyme A reductase in vitro," Bioscience, Biotechnology and Biochemistry, vol. 68, no. 2, pp. 428-432, 2004.

[20] M.-C. Rho, S. W. Lee, H. R. Park et al., "ACAT inhibition of alkamides identified in the fruits of Piper nigrum," Phytochemistry, vol. 68, no. 6, pp. 899-903, 2007.

[21] X. Zhang, L. Li, Z. Xu et al., "Investigation of the interaction of naringin palmitate with bovine serum albumin: spectroscopic analysis and molecular docking," PLoS One, vol. 8, no. 3, article e59106, 2013.

[22] N. Terasaka, A. Miyazaki, N. Kasanuki et al., "ACAT inhibitor pactimibe sulfate (CS-505) reduces and stabilizes atherosclerotic lesions by cholesterol-lowering and direct effects in apolipoprotein E-deficient mice," Atherosclerosis, vol. 190, no. 2, pp. 239-247, 2007.

[23] R. Lehner and A. Kuksis, "Biosynthesis of triacylglycerols," Progress in Lipid Research, vol. 35, no. 2, pp. 169-201, 1996.

[24] S. Cases, S. J. Smith, Y.-W. Zheng et al., "Identification of a gene encoding an acyl CoA: diacylglycerol acyltransferase, a key enzyme in triacylglycerol synthesis," Proceedings of the National Academy of Sciences of the United States of America, vol. 95, no. 22, pp. 13018-13023, 1998. 


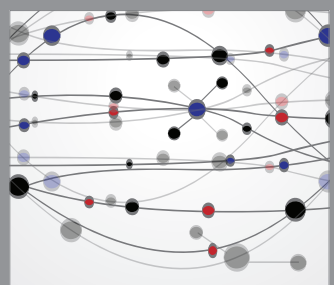

The Scientific World Journal
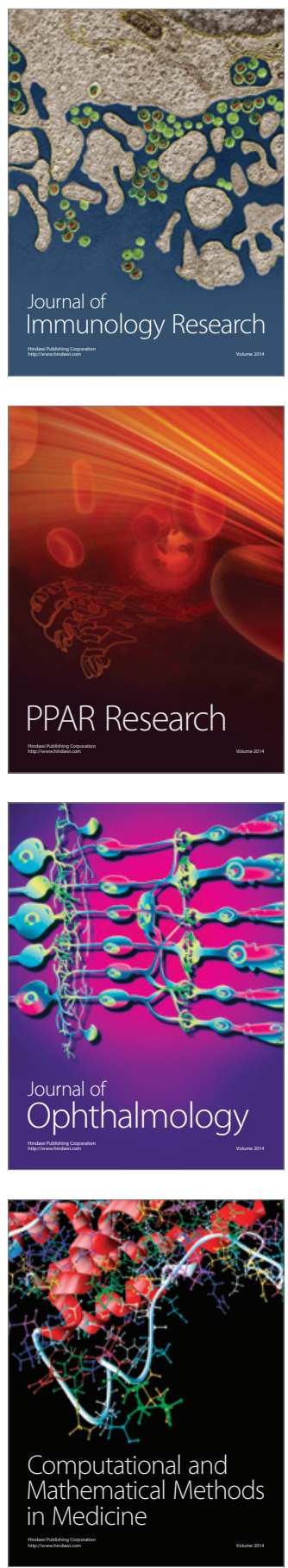

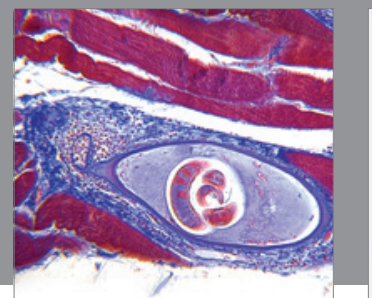

Gastroenterology

Research and Practice
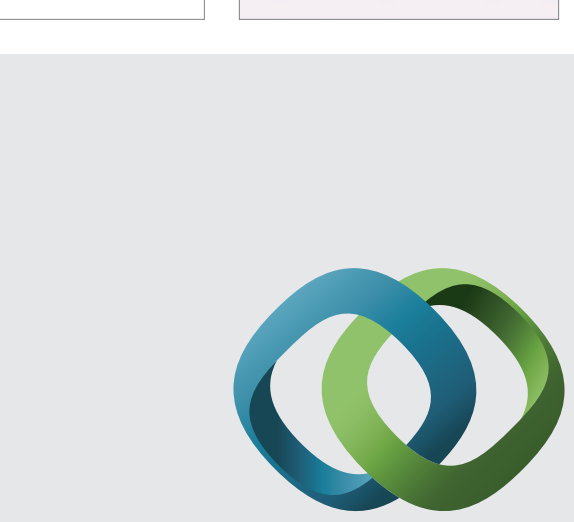

\section{Hindawi}

Submit your manuscripts at

http://www.hindawi.com
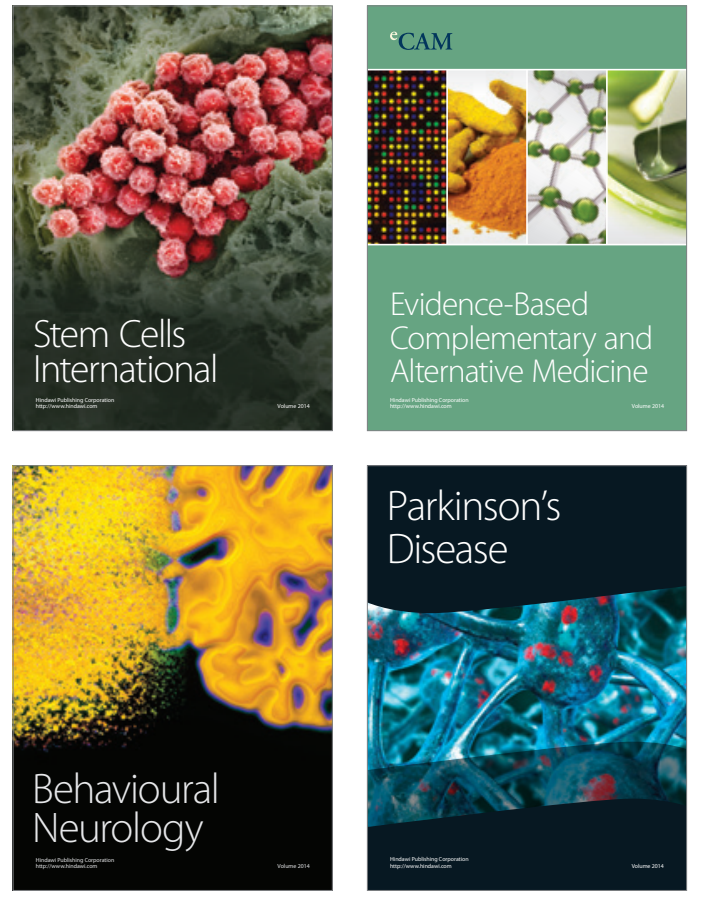
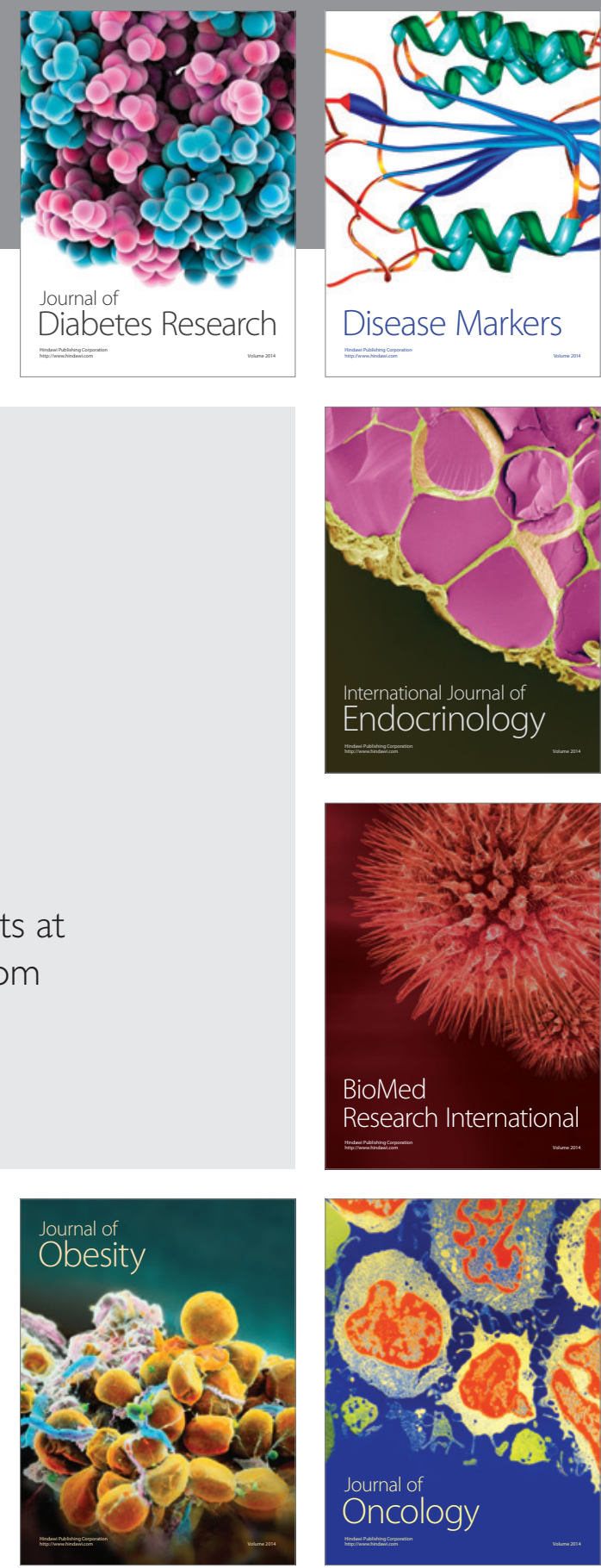

Disease Markers
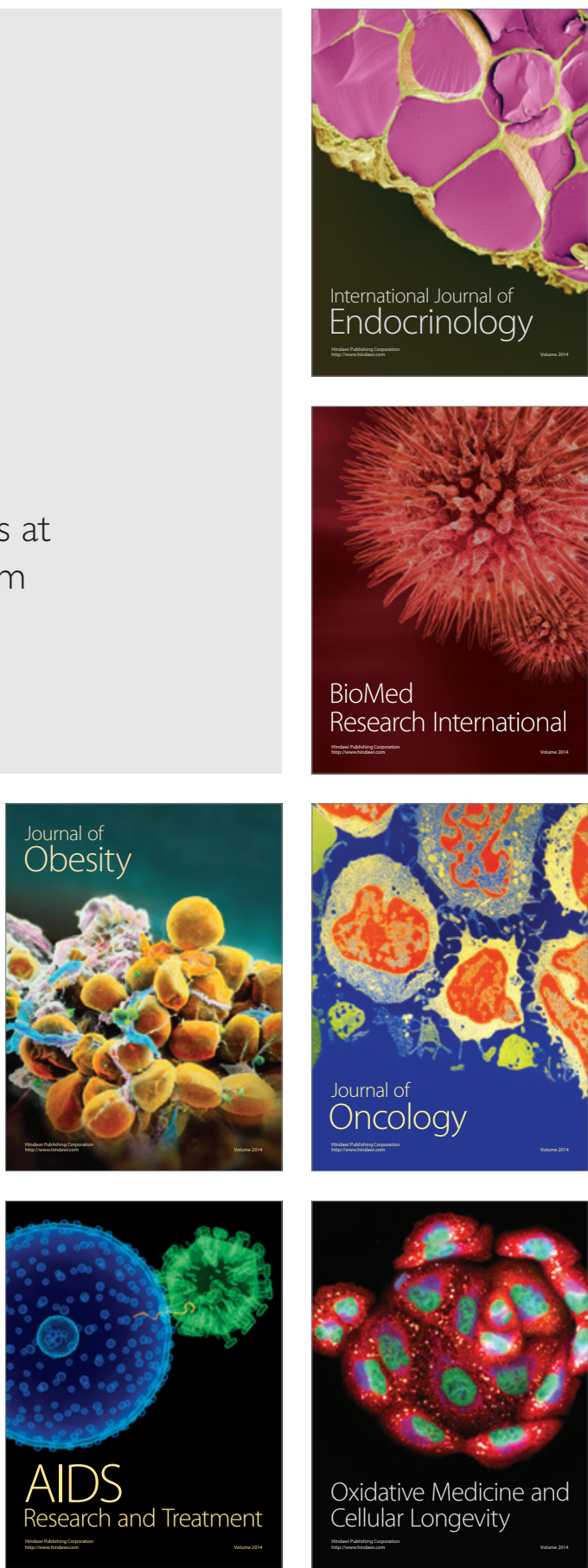